\title{
Three-dimensional reconstruction and morphological characterization of pituitary macroadenomas
}

\author{
Shou-Sen Wang, Lin Wei, Jun-Jie Jing, Shang-Ming Zhang
}

Department of Neurosurgery, Fuzhou General Hospital, Xiamen University Medical College, Fujian, China

Submitted: 3 March 2014

Accepted: 6 August 2014

Arch Med Sci 2016; 12, 3: 576-586

DOI: 10.5114 /aoms.2016.59932

Copyright @ 2016 Termedia \& Banach

\section{Abstract}

Introduction: The aim was to investigate the relationship between the tumor (clinicopathologic and radiological) characteristics and the morphological parameters of pituitary macroadenoma or giant adenoma patients using a three-dimensional (3D) reconstructed model.

Material and methods: Magnetic resoanance imaging (MRI) was performed preoperatively; tumor grade was determined by the Knosp-Steiner classification and tumor morphology by the SIPAP classification. Pituitary adenomas and adjacent structures were reconstructed three-dimensionally by volume rendering.

Results: Fifty-two and 6 patients underwent surgery via the transnasal transsphenoidal or pterional approach, respectively. Knosp-Steiner grades I to IV adenomas were observed in $5.2 \%, 25.9 \%, 22.4 \%$ and $46.6 \%$ of the patients, respectively. The $3 \mathrm{D}$ model was reconstructed in all cases with superb delineation of tumor morphology and the spatial relationship between the tumor and adjacent tissues. Pituitary adenomas were categorized into intrasellar (13.8\%), suprasellar (20.7\%), infrasellar (17.2\%), and lobulated adenomas (48.3\%). Suprasellar adenomas had the smallest $\left(2.27 \pm 3.22 \mathrm{~cm}^{3}\right)$ and lobulated adenomas the largest volume $\left(24.61 \pm 30.50 \mathrm{~cm}^{3}\right)$. Intrasellar adenomas were all functioning, while $75 \%, 60 \%$ and $60.7 \%$, respectively, of suprasellar, infrasellar and lobulated adenomas were nonfunctioning, with a significant association between tumor morphology and secretory function $(p=0.005)$.

Conclusions: Three-dimensional reconstruction of pituitary macroadenomas offers a simplified morphological classification of pituitary adenomas and may be helpful for neurosurgeons to categorize and characterize pituitary adenomas.

Key words: morphology, pituitary macroadenomas, SIPAP classification, $3 \mathrm{D}$ reconstruction, volume rendering.

\section{Introduction}

Pituitary adenomas are common neoplasms constituting $10-15 \%$ of intracranial tumors, and occur in $17-25 \%$ of the population [1]. Most pituitary adenomas are benign, but approximately one third of them are invasive, encroaching upon the bones, dura, and/or other adjacent structures [2, 3]. Delineation of adenomas inside the cavernous sinus and the relation between tumors and the internal carotid artery (ICA) may assist diagnosis, preoperative planning and intraoperative management of adenoma patients by neurosurgeons. Common imaging methods for pi-

\author{
Corresponding author: \\ Shou-Sen Wang \\ Department of Neurosurgery \\ Fuzhou General Hospital \\ Xiamen University \\ Medical College \\ 156 Road Xi'erhuanbei \\ Fuzhou \\ Fujian 350025, China \\ Phone: +86 59124937080 \\ E-mail:wshsen@126.com
}


tuitary adenomas are computed tomography (CT) and magnetic resonance imaging (MRI), which typically display images in two dimensions (2D) and are different from the three-dimensional (3D) environment in which a neurosurgeon navigates during an operation. Surgeons are required to integrate 2D images with their anatomical knowledge and surgical experience and reconstruct the spatial relations between tumors and their adjacent structures mentally. Therefore, there has been considerable interest in rendering 2D images into $3 \mathrm{D}$ images that more closely mimic the surgical environment of the brain. Image reconstruction of normal anatomic structures and lesions has been employed for surgical training and preoperative planning, including transsphenoidal surgery. Studies by Ferroli et al. [4] and Hao et al. [5] have indicated that $3 \mathrm{D}$ reconstruction of $2 \mathrm{D}$ images may assist neurosurgeons in preoperative planning by familiarizing them with the spatial relation between tumors and their adjacent structures.

On the other hand, with application of the 3D reconstructed model, the spatial morphology of pituitary adenomas and its relationship with the tumor characteristics could likely be better understood. In this study, we aimed to investigate the relationship between the tumor characteristics and the morphological parameters of pituitary adenomas by means of 3D reconstructed models. First, we investigated the clinicopathologic and radiological characteristics of 58 patients with pituitary macroadenoma or giant adenoma, such as secretion function of tumor, dura invasiveness, texture of tumor, cystic degeneration of tumor, and tumor size, and then carried out 3D reconstruction of the MRI images of these patients for morphological categorization of pituitary adenomas. Our study offers a simplified scheme of morphological classification of pituitary adenomas and may be helpful for neurosurgeons to categorize and characterize pituitary adenomas.

\section{Material and methods}

\section{Subjects}

Fifty-eight consecutive pituitary adenoma patients who sought treatment at our hospital between December 2009 and March 2012 were included in the study. These patients had pathologically proven macroadenomas or giant adenomas. The study protocol (2010-09-03) was approved by the Ethics Committee of our hospital and informed consent was not required because of the retrospective nature of this study.

\section{Radiological studies}

Magnetic resonance imaging was performed preoperatively using a 3.0-tesla MR scanner with a head birdcase transmit/receiver coil. Regular T1-weighted and T2-weighted images were used, and a gradient echo (GRE) sequence (TR/TE $1900 / 3 \mathrm{~ms}$, fov $250 \times 250, \mathrm{SL}, 1 \mathrm{~mm}$ ) was used for T1-weighted post-contrast coronal imaging perpendicular to the frontal base. Magnetic resonance imaging images were interpreted by 3 experienced radiologists independently and a consensus reading was obtained when the radiologists disagreed over grading. Knosp-Steiner classification was carried out as previously described [6] where grade 0 indicates that the tangent of the medial aspects of the intra- and supracavernous ICA is not passed; grade 1 indicates that the medial tangent is passed, but the extension does not go beyond a line drawn between the cross-sectional centers of the intra- and supracavernous ICA; grade 2 indicates that the tumor extends beyond the intercarotid line, but does not pass the tangent on the lateral aspects of the intra- and supracavernous ICA; grade 3 indicates that the tumor has extended laterally to the lateral tangent of the intra- and supracavernous ICA; grade 4 indicates that the tumor has totally encased the intracavernous carotid artery. SIPAP classification was carried out as previously described [7].

\section{Determination of tumor grades}

Tumor grade was calculated by the extent of expansion of pituitary adenoma in the suprasellar, infrasellar, parasellar, presellar and postsellar direction, and tumor grade for lateral growth of pituitary adenoma was determined according to the Knosp-Steiner classification. Pituitary adenoma was categorized by morphology according to the SIPAP classification into group I, tumor grade: 0-1, 0, 0-1, 0-1, 0 and 0; group II, tumor grade surpassed group I in only 1 direction; group III, tumor grade surpassed group I in 2 directions; group IV, tumor grade surpassed group I in at least 3 directions. For suprasellar directions, grade 0 indicated no bulging of adenoma into the suprasellar space; grade 1 indicated bulging of adenoma into the suprasellar cistern, but without reaching the optical chiasm; grade 2 indicated extension of adenoma to the optic chiasm, but without displacement of the optic chiasm; grade 3 indicated displacement and stretching to a variable degree of the optic chiasm; grade 4 indicated obstructive hydrocephalus of one or both lateral ventricles due to tumor extension. For infrasellar directions, grade 0 indicated that the sellar floor was intact; grade 1 indicated focal bulging of adenoma, which is an indirect sign of perforation of the dura and the sellar floor; grade 2 indicated tumor penetration within the sphenoid sinus. For adenomas with anterior extension, grade 0 indicated no tumor extension into the anterior cranial fossa; grade 1 indicated 
growth of adenoma into the anterior fossa. For adenoma with posterior extension, grade 0 indicated no posteroinferior growth of adenoma behind the clivus; grade 1 indicated tumor growth behind and inferior to the dorsum of the sella or clivus into the prepontine subarachnoid space.

\section{Three dimensional reconstruction of pituitary adenomas}

The scan volume included $20-30 \mathrm{~mm}$ above and below the sella turcica, and the scan data were recorded and entered into the Dextroscope workstation (Volume Interactions Co., Singapore). Window width, image intensity and image size were adjusted to sharpen the definition of tumor borders, and preliminary MRI data were then added to the virtual environment for image processing. Two experienced neurosurgeons wearing stereoscopic glasses examined the 3D virtual images in the reflection mirror in the monitor with a sensor stick and virtual light pen in each hand. The neurosurgeons manipulated the images in the virtual space and delineated the border between the tumor tissue and adjacent normal tissues by artificially tracing the tumor contour. The volume-rendering method (3DVirtuoso, Siemens AG, Forchheim, Germany) operating on a Silicon Graphics board (Silicon Graphics, Mountain View, CA) was used to reconstruct the tumor tissue. The ICA was reconstructed using threshold image segmentation. The structures of interest were colored and the images were dissected 3-dimensionally using the 3D software ReadioDexter 1.0 (Volume Interactions Co., Singapore).

\section{Morphological categorization of pituitary adenomas}

Morphological observation was carried out on $3 \mathrm{D}$ reconstructed virtual images in combination with MRI images. Pituitary adenomas were categorized into: 1 ) intrasellar adenomas ( $\leq$ KnospSteiner classification grade 2 ), which were located within the sella turcica and did not encroach upon the optic chiasm; 2) suprasellar adenomas, which extended suprasellarly and encroached upon the optic chiasm but did not penetrate the sellar floor; 3) infrasellar adenomas, which extended inferiorly and penetrated the sellar floor to reach the sphenoid sinus and beyond; 4) lobulated adenomas ( $\geq$ Knosp-Steiner classification grade 3 ), which extended suprasellarly in at least 2 lobules or encroached upon the clivus.

\section{Surgical treatment}

All adenomas except giant inverse "8" adenomas and giant suprasellar lobulated adenomas were accessed by the transnasal transsphenoidal approach [8, 9]. Fifty-two patients underwent surgery via the transnasal transsphenoidal approach and 6 patients via the pterional approach. Tumor texture was classified as soft, medium or hard as described by Qi et al. [10].

\section{Immunohistochemistry}

In cases undergoing transnasal transsphenoidal surgery, after dissection of the sellar floor, the dura was partially excised for pathological examination. The sections were immunostained with antibodies against prolactin (PRL), growth hormone $(\mathrm{GH})$, adrenocorticotropic hormone $(\mathrm{ACTH})$, follicle-stimulating hormone (FSH), and luteinizing hormone (LH) (all from Santa Cruz Biotechnology, Santa Cruz, CA). Five slices were selected randomly for analysis from each patient, and 5 visual fields were analyzed in each slide. Null cell pituitary adenoma and LH/FSH-positive pituitary adenoma had no clinical manifestations and were considered nonfunctioning adenomas, while other hormone-positive adenomas were considered functioning adenomas.

\section{Statistical analysis}

All continuous data were expressed as median (interquartile range), and the Kruskal-Wallis test was used to test the differences among groups. As long as a significant result was revealed by the Kruskal-Wallis test, the Mann-Whitney $U$ test was then carried out for post-hoc tests. The Mann-Whitney $U$ test was also implemented for comparison between 2 groups. Categorical variables were expressed as numbers (percentage) and examined by Fisher's exact test. The value of $p<0.05$ was considered statistically significant. The significance level was adjusted to 0.01 when post-hoc tests were implemented less than 10 times; the level was further adjusted to 0.005 when post-hoc tests were needed more than 10 times. All statistical tests were two-sided and were performed using the SPSS software (version 15.0, SPSS Inc, Chicago, IL).

\section{Results}

\section{Patient demographic and baseline characteristics}

Fifty-eight patients with pituitary adenoma were included in the study. Patient demographic and baseline characteristics are shown in Table I. There were 33 males and 25 females, and their median age was 47.5 years (range: 7 to 73 years). Complete tumor resection was achieved in $58.6 \%(34 / 58)$ of the patients and subtotal resection in $41.4 \%(24 / 58)$ of the patients. Meningeal involvement was observed in $77.8 \%(42 / 54)$ of 
the patients. Pathological examination of surgical specimens showed PRL-positive adenomas in $7(12.1 \%)$ cases, GH-positive adenomas in 4 (6.9\%) cases, ACTH-positive adenomas in 2 (3.5\%) cases, FSH-positive adenomas in 17 (29.3\%) cases, TSH-positive adenomas in $3(5.2 \%)$ cases, the mixed type in $10(17.2 \%)$ cases, and the null cell type in 15 (25.9\%) cases. Cystic changes were observed in half of the cases (29/58).

We further analyzed the correlation between clinicopathologic parameters and tumor volume in 58 pituitary adenoma patients (Table II). Males were prone to have greater tumor size than females $(p=0.032)$. We observed no significant difference in tumor volume between cystic adenomas and non-cystic adenomas $(p>0.05)$. Furthermore, there was no significant difference in tumor volume among adenomas of different textures $(p>0.05)$. Among 52 patients undergoing transnasal transsphenoidal resection, totally resected adenomas were significantly smaller in size (median $6.4 \mathrm{~cm}^{3}$ ) than subtotally resected adenomas (median $\left.14.7 \mathrm{~cm}^{3}\right)(p<0.001)$. Moreover, patients with FSH-secreting adenomas were prone to have greater tumor size than patients with plurihormonal adenomas $(p=0.004)$, while no difference in tumor volume was observed among adenomas of other pathologic types.

\section{SIPAP classification of pituitary adenomas}

Three (5.2\%) patients were classified as SIPAP grade I, $15(25.9 \%)$ as grade II, $13(22.4 \%)$ as grade III and 27 (46.6\%) as grade IV. Patient demographic and disease characteristics are listed in Table III by SIPAP classification. There was no significant difference in age $(p=0.355)$ among pituitary adenomas of different SIPAP classifications. All 3 patients in grade I were female, while grade $\|$ or above patients were mostly male (about 60\%). There was no difference in sex among different grades of pituitary adenomas $(p=0.283)$. There was a significant difference in volume among pituitary adenomas of different SIPAP classifications $(p<0.001)$. The volume of grade IV adenomas was larger than that of other grades of adenoma ( $p_{\text {I-vs.IV }}=0.010, p_{\text {II-vs.IV }}=0.004$, and $p_{\text {III-vs.IV }}=0.0002$ ). Furthermore, no significant association was found between the functioning status of pituitary adenomas and SIPAP classification $(p=0.229)$. SIPAP grade I, II and IV adenomas had fewer cases of medium texture, but no significant association was found between tumor texture and SIPAP classification ( $p=0.972)$. Cystic changes were observed in $33.3 \%$ of grade I adenomas, $46.7 \%$ of grade II adenomas, $53.9 \%$ of grade III adenomas and $51.9 \%$ of grade IV adenomas. No significant association was found between cystic changes and SIPAP classification $(p=0.999)$. Ad-
Table I. Patient demographic and baseline characteristics of study participants $(n=58)$

\begin{tabular}{|c|c|}
\hline Parameter & Results \\
\hline Age, mean \pm SD [years] & $45.7 \pm 14.8$ \\
\hline Male gender, $n(\%)$ & $33(56.9)$ \\
\hline \multicolumn{2}{|l|}{ Surgical approach, $n(\%)$ : } \\
\hline Transnasal transsphenoidal & $52(89.7)$ \\
\hline Pterional & $6(10.3)$ \\
\hline Tumor size, mean (range) $\left[\mathrm{cm}^{3}\right]$ & $16.5(0.97-137.7)$ \\
\hline \multicolumn{2}{|l|}{ Tumor resection, $n$ (\%): } \\
\hline Complete & $34(58.6)$ \\
\hline Subtotal & $24(41.4)$ \\
\hline \multicolumn{2}{|l|}{ Cystic changes, $n(\%)$ : } \\
\hline Yes & $29(50)$ \\
\hline No & $29(50)$ \\
\hline \multicolumn{2}{|l|}{ Dura involvement $(n=54), n(\%)$ : } \\
\hline Yes & $42(77.8)$ \\
\hline No & $12(22.2)$ \\
\hline \multicolumn{2}{|l|}{ Tumor texture, $n(\%)$ : } \\
\hline Soft & $18(31.0)$ \\
\hline Medium & $12(20.7)$ \\
\hline Hard & $28(48.3)$ \\
\hline \multicolumn{2}{|l|}{ Pathological type, $n(\%)$ : } \\
\hline PRL & $7(12.1)$ \\
\hline FSH & $17(29.3)$ \\
\hline $\mathrm{GH}$ & $4(6.9)$ \\
\hline ACTH & $2(3.5)$ \\
\hline TSH & $3(5.2)$ \\
\hline Mixed & $10(17.2)$ \\
\hline Null cell type & $15(25.9)$ \\
\hline \multicolumn{2}{|l|}{ Morphological classification, $n(\%)$ : } \\
\hline Intrasellar adenoma & $8(13.8)$ \\
\hline Suprasellar adenoma & $12(20.7)$ \\
\hline Infrasellar adenoma & $10(17.2)$ \\
\hline Lobulated adenoma & $28(48.3)$ \\
\hline
\end{tabular}

ditionally, meningeal invasion was noted in $66.7 \%$ of grade I adenomas, $85.7 \%$ of grade II adenomas, $76.9 \%$ of grade III adenomas, and $75.0 \%$ of grade IV adenomas. There was no significant difference in meningeal invasion among pituitary adenomas of different SIPAP classifications ( $p=0.905$ ). Among 52 patients receiving transnasal transsphenoidal resection, complete tumor resection was completed in 100\% (3/3) of grade I adenoma patients, $73.3 \%(11 / 15)$ of grade II adenoma patients, $69.2 \%$ (9/12) of grade III adenoma patients and $40.7 \%$ (11/27) of grade IV adenoma patients, indicating a lower complete resection rate with increased SIPAP grade. However, no apparent association was observed between tumor resection rate and SIPAP classification $(p=0.061)$. 
Table II. Correlation of patient demographic and clinicopathological characteristics and tumor volume

\begin{tabular}{|c|c|c|c|c|}
\hline Parameter & No. of cases & Tumor volume $\left[\mathrm{cm}^{3}\right]$ & $P$-value & Post-hoc test $^{1}$ \\
\hline Gender: & & & $0.032^{*}$ & $M>F$ \\
\hline Male & 33 & $10.9(7.9,17.5)$ & & \\
\hline Female & 25 & $6.1(3.1,19.0)$ & & \\
\hline Cystic tumor: & & & 0.074 & \\
\hline No & 29 & $7.5(3.4,14.4)$ & & \\
\hline Yes & 29 & $10.4(7.9,19.7)$ & & \\
\hline Meningeal invasion: & & & 0.556 & \\
\hline $\mathrm{No}^{2}$ & 12 & $11.2(4.7,23.5)$ & & \\
\hline Yes & 42 & $10.1(4.2,14.3)$ & & \\
\hline Tumor texture: & & & 0.550 & \\
\hline Soft & 18 & $7.9(4.6,12.4)$ & & \\
\hline Medium & 12 & $12.0(7.5,14.7)$ & & \\
\hline Hard & 28 & $10.8(4.0,20.2)$ & & \\
\hline Pathological type: & & & $0.001^{*}$ & FSH $>$ Pluri \\
\hline Null cell & 15 & $10.2(6.1,19.7)$ & & \\
\hline Prolactin & 7 & $9.0(2.5,52.1)$ & & \\
\hline $\mathrm{FSH}$ & 17 & $13.7(10.9,15.0)$ & & \\
\hline $\mathrm{TSH}$ & 3 & $38.2(33.4,89.7)$ & & \\
\hline $\mathrm{GH}$ & 4 & $4.3(3.9,5.5)$ & & \\
\hline Mixed & 10 & $3.4(3.9,5.5)$ & & \\
\hline ACTH & 2 & $4.8(1.7,7.9)$ & & \\
\hline Tumor resection: & & & $<0.001^{*}$ & $P>C$ \\
\hline Complete & 34 & $6.4(3.7,10.6)$ & & \\
\hline Subtotal & 24 & $14.7(9.7,30.7)$ & & \\
\hline \multicolumn{5}{|c|}{$\begin{array}{l}\text { ACTH - adrenocorticotropic hormone, FSH-follicle-stimulating hormone, GH-growth hormone, PRL-prolactin, TSH-thyroid-stimulating } \\
\text { hormone. Data are presented as median (interquartile range) and were tested by Mann-Whitney U test for pair-wise comparison or } \\
\text { Kruskal-Wallis test. }{ }^{1} \text { Mann-Whitney } U \text { test was performed for post-hoc test. Significant level was adjusted to } 0.01 \text { for variables with the } \\
\text { number of post-hoc tests }<10 \text { or to } 0.005 \text { for variables with the number of post-hoc tests }>10 .{ }^{2} \text { There were } 4 \text { missing data found in patients } \\
\text { without meningeal invasion. *Indicates significant difference among various groups, } p<0.05 \text {. }\end{array}$} \\
\hline
\end{tabular}

\section{Three-dimensional reconstruction}

A three-dimensional reconstruction model of pituitary macroadenoma and giant adenoma was established using the Dextroscope Image Workstation, and bilateral ICA was successfully reconstructed in all cases (Figure 1). For each case, the tumor was clearly visualized and the tumor border was distinctly demarcated. The 3D reconstructed images delineated tumor morphology and revealed the spatial relationship between tumor and adjacent tissues. In some cases, normal pituitary tissues and tumor tissues were reconstructed, and coloring of tumors and the ICA clearly defined the spatial relationship between tumor and the ICA (Figure 2).

\section{Patient demographic and disease characteristics by morphological types}

Intrasellar adenomas were found in 8 (13.8\%) cases, suprasellar adenomas in 12 (20.7\%) cases, infrasellar adenomas in 10 (17.2\%) cases, and lob- ulated adenomas in 28 (48.3\%) cases (Figure 3). Patient demographic and disease characteristics are listed in Table IV by morphological types. There was no significant difference in age ( $p=0.632)$ or sex $(p=0.107)$ among the four types of adenomas. Intrasellar adenoma was significantly different in size from infrasellar and lobulated adenomas ( $p_{\text {intrasellar-vs.-infrasellar }}=0.001, p_{\text {intrasellar-vs.lobulated }}$ $=0.004)$. Infrasellar adenoma showed no statistically significant difference in size from suprasellar adenomas and lobulated adenomas $(p>0.01)$. Additionally, we found no significant difference in tumor texture among the four types of adenomas $(p>0.05)$. Most patients ( $>65 \%$ ) had meningeal invasion regardless of tumor shape. No statistically significant association was noted between meningeal invasion and tumor morphology $(p=0.643)$. Cystic changes were seen more frequently in infrasellar adenomas $(8 / 10,80 \%)$ than other types of adenomas, but no statistically significant association was noted between cystic changes and tumor morphology $(p=0.226)$. Among 52 patients 
Table III. Correlation of patient demographic and disease characteristics and SIPAP classification

\begin{tabular}{|lccccc|}
\hline Parameter & $\begin{array}{c}\text { I } \\
(n=3)\end{array}$ & $\begin{array}{c}\text { II } \\
(n=15)\end{array}$ & $\begin{array}{c}\text { III } \\
(n=13)\end{array}$ & $\begin{array}{c}\text { IV } \\
(n=27)\end{array}$ & $P_{\text {-value }}{ }^{1}$ \\
\hline Age, median (range) [years] & $24(18-48)$ & $48(36-62)$ & $47(36-54)$ & $48(37-59)$ & 0.355 \\
\hline $\begin{array}{l}\text { Tumor volume, median } \\
\left.\text { (interquartile range) [cm }{ }^{3}\right]\end{array}$ & $2.4(1.8-2.5)^{\dagger}$ & $4.6(3.7-7.9)^{\dagger}$ & $8.3(4.8-11.2)^{\dagger}$ & $17.5(10.6-33.4)$ & $<0.001^{*}$ \\
\hline Male gender, $n(\%)$ & $0(0.0)$ & $9(60.0)$ & $8(61.5)$ & $16(59.3)$ & 0.283 \\
\hline $\begin{array}{l}\text { Pathological type, } n(\%): \\
\text { Null cell }\end{array}$ & $0(0.0)$ & $4(26.7)$ & $4(30.8)$ & $7(25.9)$ & 0.229 \\
\hline PRL & $2(66.7)$ & $1(6.7)$ & $1(7.7)$ & $3(11.1)$ & \\
\hline FSH & $0(0.0)$ & $3(20.0)$ & $4(30.8)$ & $10(37.0)$ & \\
\hline TSH & $0(0.0)$ & $0(0.0)$ & $0(0.0)$ & $3(11.1)$ & \\
\hline GH & $0(0.0)$ & $2(13.3)$ & $2(15.4)$ & $0(0.0)$ & \\
\hline Plurihormonal & $1(33.3)$ & $4(26.7)$ & $1(7.7)$ & $4(14.8)$ & \\
\hline ACTH & $0(0.0)$ & $1(6.7)$ & $1(7.7)$ & $0(0.0)$ & \\
\hline Texture of tumor, $n(\%):$ & $1(33.3)$ & $5(33.3)$ & $3(23.1)$ & $9(33.3)$ & \\
\hline Soft & $0(0.0)$ & $3(20.0)$ & $4(30.8)$ & $5(18.5)$ & \\
\hline Medium & $2(66.7)$ & $7(46.7)$ & $6(46.2)$ & $13(48.2)$ & \\
\hline Hard & $1(33.3)$ & $7(46.7)$ & $7(53.9)$ & $14(51.9)$ & 0.999 \\
\hline Cystic changes, $n(\%)$ & $2(66.7)$ & $12(85.7)$ & $10(76.9)$ & $18(75.0)$ & 0.905 \\
\hline Meningeal invasion, $n(\%)^{2}$ & $3(100.0)$ & $11(73.3)$ & $9(69.2)$ & $11(40.7)$ & 0.061 \\
\hline Tumor resection, $n(\%):$ & $0(0.0)$ & $4(26.7)$ & $4(30.8)$ & $16(59.3)$ & \\
\hline Complete & & & & \\
\hline Subtotal & & & & \\
\hline
\end{tabular}

ACTH - adrenocorticotropic hormone, FSH - follicle-stimulating hormone, GH - growth hormone, PRL - prolactin, SIPAP - suprasellar infrasellar parasellar anterior posterior, TSH - thyroid-stimulating hormone. ${ }^{1}$-values were derived from Kruskal-Wallis test for continuous variables and Fisher's exact test for categorical variables. ${ }^{2}$ There was 1 missing datum in grade II and 3 missing data in grade IV. * Indicates significant difference among various groups, $p<0.05{ }^{\dagger}{ }^{\dagger}$ Indicates significant difference from group IV, $p<0.01$.

who underwent single transnasal transsphenoidal resection, complete tumor resection was completed in $100 \%(8 / 8)$ of intrasellar adenoma patients, $58.3 \%(7 / 12)$ of suprasellar adenoma patients, $70.0 \%(7 / 10)$ of infrasellar adenoma patients and $42.9 \%(12 / 28)$ of lobulated adenoma patients. There was a statistically significant difference between lobulated and intrasellar adenoma patients $(p=0.005)$. Furthermore, a significant association between tumor morphology and rate of complete tumor resection was found ( $p=0.039)$. Relative to lobulated adenoma, a higher complete tumor resection rate was found in intrasellar adenoma patients $(p=0.010)$.

Intrasellar adenomas were all functioning (100\%), $75 \%$ of suprasellar adenomas were nonfunctioning, $60 \%$ of infrasellar adenomas were nonfunctioning, and $60.7 \%$ of lobulated adenomas were nonfunctioning. A significant association was found between tumor morphology and the secretory function of pituitary adenomas $(p=0.004)$. The distribution of pathological types among the 4 groups was significantly different as well. A higher percentage of FSH-secreting suprasellar adenomas and lobulated adenomas was found compared with intrasellar adenomas, while a larger percentage of plurihormone intrasellar adenomas was observed compared with lobulated adenomas ( $p_{\text {intrasellar-vs.-suprasellar }}=0.003, p_{\text {intrasellar-vs.-lobulated }}$ $=0.001)$. Moreover, the percentage of null cell adenomas was significantly higher in suprasellar and lobulated adenomas than that of intrasellar adenomas.

\section{Discussion}

Transnasal transsphenoidal surgery offers the least traumatic route to the sella turcica for resection of pituitary adenomas and is generally safe in the hands of experienced neurosurgeons. Because the adjacent neurovascular structures may be inadvertently damaged if inappropriately accessed, it is very important for a neurosurgeon to have detailed knowledge of the 3D anatomical features of the tumor and its adjacent structures. In previous reports, tumor volume has been largely estimated by measuring the diameter of the tumor [11], or calculated by the formula: $(a \times b \times c) \pi / 6$ [12], where $a, b$, and $c$ represent the three dimensions of the tumor. However, pituitary adenomas are known to be varied in shape, and these two methods could only offer a rough estimate of tumor 

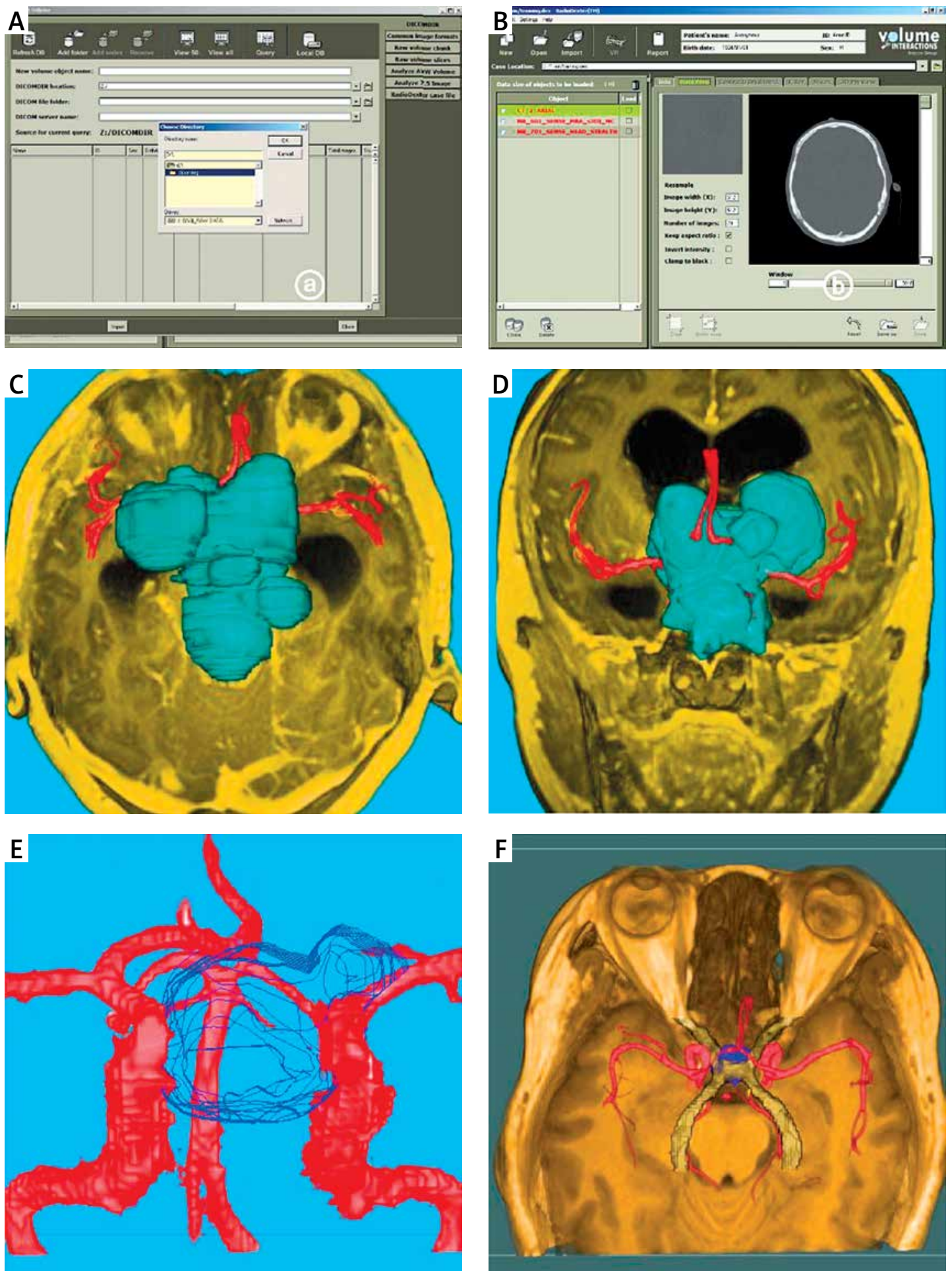

Figure 1. Three-dimensional reconstruction of a lobulated pituitary macroadenoma. The original files were imported into the VR station (A) and data were prepared (B). C, D - Lobulated adenoma (light blue), the internal carotid artery (ICA) (red) and adjacent soft tissues were reconstructed. E-Tumor was outlined (blue) by artificial tracing of the contours of the tumor. F - The 3D reconstructed image of the ICA and the optic chiasma

volume [13]. Surgeons often have to rely on 2D images from $\mathrm{CT}$ and MRI to mentally reconstruct the spatial relations between tumors and their adjacent structures by integrating their anatomical knowledge and surgical experience. Based on the
2D image of MRI, it is difficult for neurosurgeons to determine the spatial morphologies of pituitary adenomas with irregular shape, especially the protuberances, which may be useful for predicting the invasive characteristics of pituitary adenomas. 

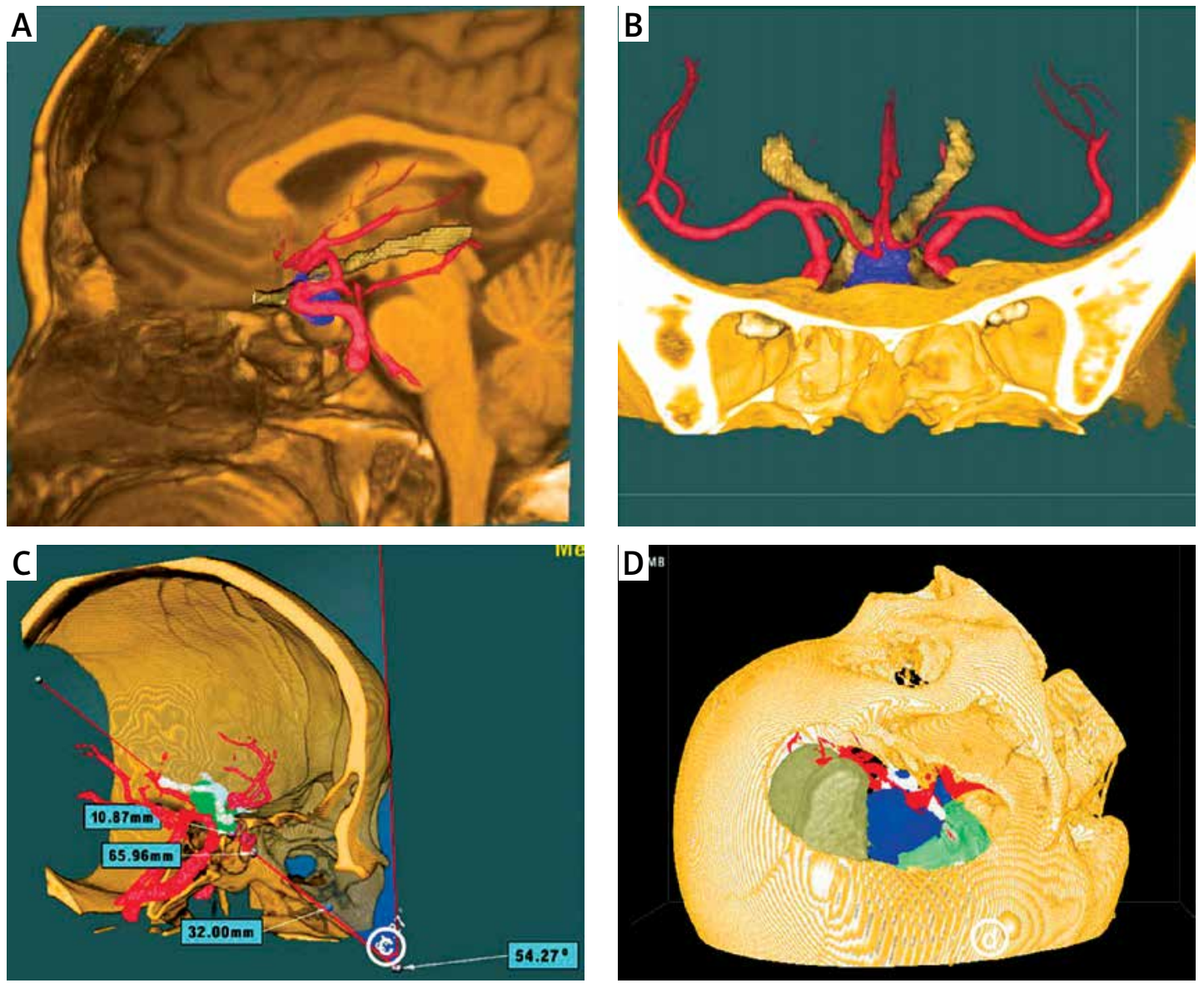

Figure 2. Three-dimensional reconstruction of a pituitary macroadenoma. A - The 3D reconstructed image of pituitary macroadenoma (blue), the ICA (light red) and the optic chiasma (light brown). B - The 3D reconstructed image of pituitary macroadenoma (blue), the ICA (light red), the optic chiasma (light brown) and the cranium. C - Virtual determination of distance and angle via the transnasal transsphenoidal approach. D - Virtual simulation of the transcranial approach

There has been growing interest in rendering 2D images of brain tumors and adjacent structures into 3D images that more closely mimic the surgical environment of the brain, as CT and MRI images are less desirable in defining the geometric shape of anatomic structures and delineating the spatial relations between tumors and adjacent structures. In the current study, we reconstructed 3D images of pituitary adenomas and adjacent structures from MRI data by volume rendering, which projects each voxel directly onto the picture plane and consequently the 3D structure. Threshold segmentation, which separates the region of interests from adjacent regions for image augmentation and subsequent quantitative analysis of the target region, is still of great importance in image processing [14]. Currently, there is no software for effective threshold segmentation of the region of interest. Here, we delineated the border between the tumor tissue and adjacent normal tissue by artificially tracing the tumor contour. Because we used enhanced MRI scanning, which well demarcated the tumor border and adjacent tissues, arti- ficial tracing could accurately demarcate the actual tumor border. In images in DICOM format, a precise value is assigned to the length represented by each voxel. Dextroscope Image Workstation can sum up the volume represented by each voxel in the $3 D$ model to derive a relatively precise value of the total volume. This method is similar to that proposed by Ertekin, but is more precise due to direct processing of DICOM documents. Here, we successfully established the 3D model of pituitary macroadenomas and giant adenomas using Dextroscope Image Workstation, and the 3D reconstructed images could delineate tumor morphology and reveal the spatial relationship between the tumor and adjacent tissues. Furthermore, the ICA was successfully reconstructed in all the patients and coloring of tumors and the ICA could clearly define the spatial relationship between tumor and the ICA. The use of continuous scanning of MRI at a slice thickness of $1 \mathrm{~mm}$ in our study ensured the image quality to a great extent.

Preoperative 3D visualization of the morphology of pituitary adenomas assists in preoperative 

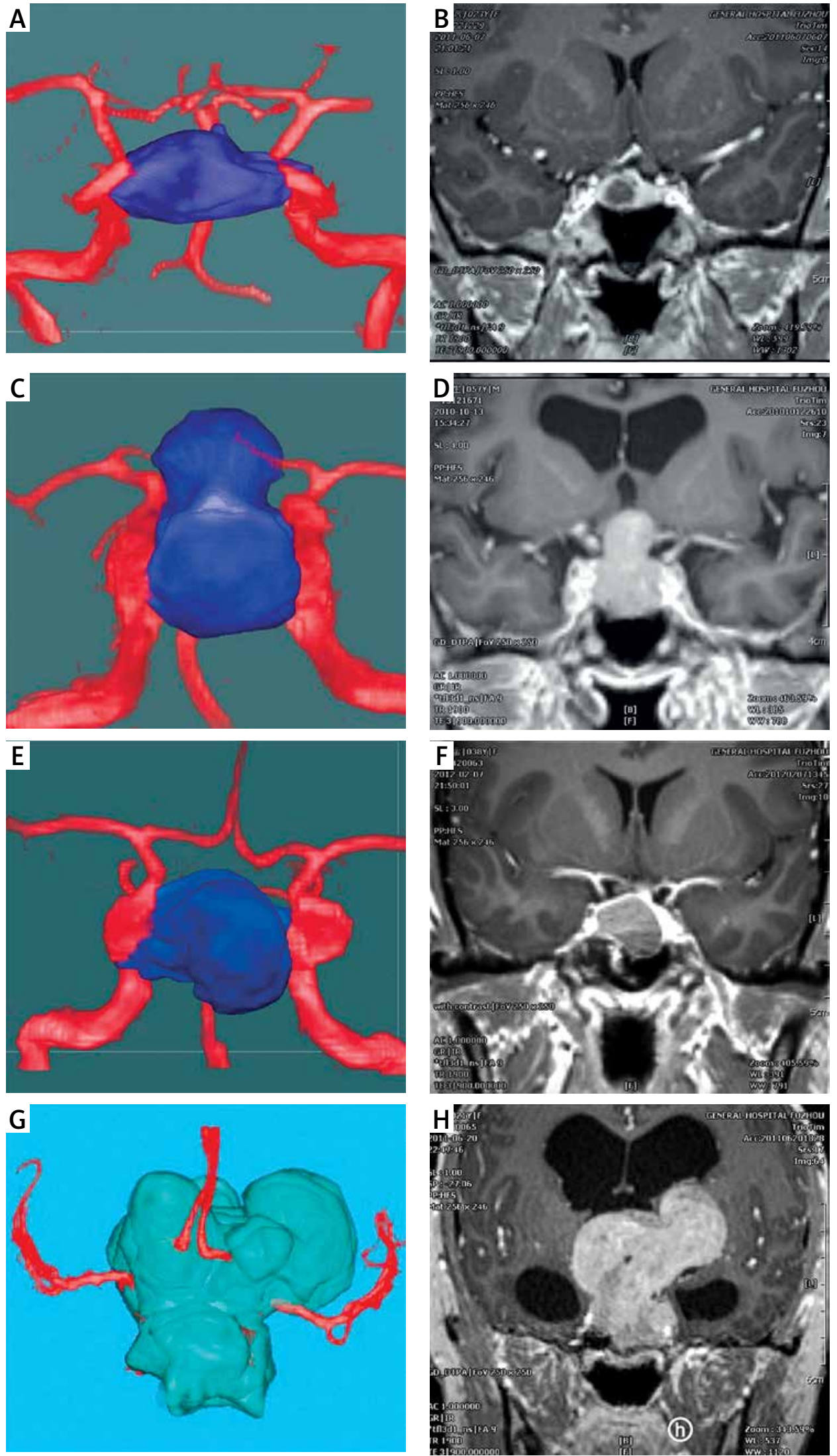

Figure 3. Morphological classification of pituitary adenomas. A, B - Intrasellar adenoma, no extension of the tumor; C, D - suprasellar adenoma, the tumor bulges superiorly into the suprasellar cistern; E, F - infrasellar adenoma, the tumor penetrates inferiorly into the sphenoid sinus; $\mathbf{G}, \mathbf{H}$ - lobulated adenoma, the tumor bulges upwards 
Table IV. Patient demographic and disease characteristics by morphological type

\begin{tabular}{|c|c|c|c|c|c|}
\hline Parameter & $\begin{array}{l}\text { Intrasellar } \\
\text { adenoma } \\
(n=8)\end{array}$ & $\begin{array}{l}\text { Suprasellar } \\
\text { adenoma } \\
(n=12)\end{array}$ & $\begin{array}{l}\text { Infrasellar } \\
\text { adenoma } \\
(n=10)\end{array}$ & $\begin{array}{l}\text { Lobulated } \\
\text { adenoma } \\
(n=28)\end{array}$ & $P$-value ${ }^{1}$ \\
\hline Age, median (range) [years] & $42.0(25.5-55)$ & $52.5(44.5-60.5)$ & $46.5(36.0-54.0)$ & $47.5(36.5-55.5)$ & 0.632 \\
\hline \multicolumn{6}{|l|}{ Gender, $n(\%)$ : } \\
\hline Male & $2(25.0)$ & $8(66.7)$ & $4(40.0)$ & 19 (67.9) & 0.107 \\
\hline Female & $6(75.0)$ & $4(33.3)$ & $6(60.0)$ & $9(32.1)$ & \\
\hline $\begin{array}{l}\text { Tumor volume, median } \\
\text { (interquartile range) }\left[\mathrm{cm}^{3}\right]\end{array}$ & $3.4(2.5-4.3)$ & $7.9(3.3-14.4)$ & $10.4(7.9-20.6)^{\dagger}$ & $11.8(8.4-29.8)^{\dagger}$ & $0.001^{*}$ \\
\hline Tumor texture, $n(\%)$ : & & & & & 0.823 \\
\hline Soft & $2(25.0)$ & $4(33.3)$ & $5(50.0)$ & $7(25.0)$ & \\
\hline Medium & $1(12.5)$ & $3(25.3)$ & $1(10.0)$ & $7(25.0)$ & \\
\hline Hard & $5(62.5)$ & $5(41.7)$ & $4(40.0)$ & $14(50.0)$ & \\
\hline Pathologic type, $n$ (\%): & & & & & $0.004^{*}$ \\
\hline Null cell & $0(0.0)$ & $3(25.0)^{\dagger}$ & $5(50.0)$ & $7(25.0)^{\dagger}$ & \\
\hline PRL & $3(37.5)$ & $0(0.0)$ & $0(0.0)$ & $4(14.3)$ & \\
\hline FSH & $0(0.0)$ & $6(50.0)$ & $1(10.0)$ & $10(35.7)$ & \\
\hline TSH & $0(0.0)$ & $0(0.0)$ & $0(0.0)$ & $3(10.7)$ & \\
\hline $\mathrm{GH}$ & $2(25.0)$ & $0(0.0)$ & $1(10.0)$ & $1(3.6)$ & \\
\hline Plurihormonal & $3(37.5)$ & $3(25.0)^{\dagger}$ & $3(30.0)$ & $1(3.6)^{\dagger}$ & \\
\hline ACTH & $0(0.0)$ & $0(0.0)$ & $0(0.0)$ & $2(7.1)$ & \\
\hline Functional pathology, $n$ (\%): & & & & & $0.005^{*}$ \\
\hline Functional $^{2}$ & $8(100.0)$ & $3(25.0)^{\dagger}$ & $4(40.0)^{\dagger}$ & $11(39.3)^{\dagger}$ & \\
\hline Non-functional & $0(0.0)$ & $9(75.0)$ & $6(60.0)$ & $17(60.7)$ & \\
\hline \multicolumn{6}{|l|}{ Meningeal invasion, $n(\%):^{3}$} \\
\hline Yes & $5(71.4)$ & $8(66.7)$ & $9(90.0)$ & $20(80.0)$ & 0.643 \\
\hline No & $2(28.6)$ & $4(33.3)$ & $1(10.0)$ & $5(20.0)$ & \\
\hline \multicolumn{6}{|l|}{ Cystic changes, $n$ (\%): } \\
\hline Yes & $3(37.5)$ & $5(41.7)$ & $8(80.0)$ & $13(46.4)$ & 0.226 \\
\hline No & $5(22.5)$ & $7(58.3)$ & $2(20.0)$ & $15(53.6)$ & \\
\hline Tumor resection: & & & & & $0.022^{*}$ \\
\hline Complete & $8(100.0)$ & $7(58.3)$ & $7(70.0)$ & $12(42.9)^{\dagger}$ & \\
\hline Subtotal & $0(0.0)$ & $5(41.7)$ & $3(30.0)$ & $16(57.1)$ & \\
\hline
\end{tabular}

ACTH-adrenocorticotropic hormone, FSH - follicle-stimulating hormone, GH - growth hormone, PRL-prolactin, TSH-thyroid-stimulating hormone. ${ }^{1} P$-values were derived from Kruskal-Wallis test for continuous variables and Fisher's exact test for categorical variables. ${ }^{2} N u l l$ cell and FSH were defined as functional type and others were defined as non-functional type. ${ }^{3}$ There was 1 missing datum in intrasellar adenoma and 3 missing data in lobulated adenoma. ${ }^{*}$ ndicates significant difference among various groups, $p<0.05 .{ }^{\dagger}$ Denotes significantly different from intrasellar, $p<0.01$ (or $<0.005$ ).

planning and familiarizes neurosurgeons with the 3D environment in which a neurosurgeon navigates during an operation. Currently, there is no report on the detailed morphology of pituitary adenomas. If pituitary tumors were categorized morphologically by different patterns of tumor growth in 6 directions or their combinations, the classification would be extremely cumbersome and hamper clinical analysis. Through detailed analysis of MRI data and 3D reconstructed virtual images, we developed a simplified scheme of morphological classification: 1) intrasellar adenomas, 2) suprasellar adenomas, 3) infrasellar adenomas, and 4) lobulated adenomas. We found that lobulated adenomas accounted for approximately half of our patients. Intrasellar adenomas were significantly smaller than the other three types of adenomas, and suprasellar adenomas were significantly smaller than lobulated adenomas. Additionally, intrasellar adenomas were more likely to be completely resected than lobulated adenomas. However, we found no significant difference in tumor texture, meningeal invasion, or cystic changes among the four types of adenomas. Furthermore, suprasellar adenomas and lobulated adenomas were more likely to be FSH positive 
than intrasellar adenomas, while intrasellar adenomas were more likely to be positive for multiple hormones than lobulated adenomas. Moreover, null cell adenomas were more likely to be present in suprasellar and lobulated adenomas than intrasellar adenomas.

The current study also has certain limitations. Dextroscope Image Workstation is expensive and $3 \mathrm{D}$ reconstruction of pituitary adenomas is time consuming compared with other methods. In addition, currently there is no software for effective threshold segmentation, and artificial tracing of the tumor contour has to be adopted, which is subject to human error. Furthermore, our study cohort was limited in size and the current findings need to be confirmed by similar studies involving larger patient populations.

In conclusion, we have established a reconstructed 3D model of pituitary macroadenomas or giant adenomas and developed a simplified scheme of morphological classification of pituitary adenomas based on the integrated analysis of the 3D model and MRI data. Our 3D model could offer neurosurgeons a virtual environment that mimics the 3D environment in which a neurosurgeon navigates during an operation, thus greatly facilitating preoperative planning. Furthermore, our simplified scheme of morphological classification of pituitary adenomas may offer neurosurgeons a simplified system to categorize and characterize pituitary adenomas.

\section{Conflict of interest}

The authors declare no conflict of interest.

\section{References}

1. Asa SL, Ezzat S. The pathogenesis of pituitary tumours. Nat Rev Cancer 2002; 2: 836-49.

2. Scheithauer BW, Kovacs KT, Laws ER Jr, Randall RV. Pathology of invasive pituitary tumors with special reference to functional classification. J Neurosurg 1986; 65: 733-44.

3. Colao A, Grasso LF, Pivonello R, Lombardi G. Therapy of aggressive pituitary tumors. Expert Opin Pharmacother 2011; 12: 1561-70.

4. Ferroli P, Tringale G, Acerbi F, Aquino D, Franzini A, Brogg G. Brain surgery in a stereoscopic virtual reality environment: a single institution's experience with 100 cases. Neurosurgery 2010; 67 (ONS Suppl. 1): ons 79-84.

5. Hao B, Zhou X, Jin A, et al. Preliminary exploration of three dimensional reconstruction of MRI images of human subthalamic nucleus. Chin J Neurosurg 2010; 26: 548-51.

6. Knosp E, Steiner E, Kitz K, Matula C. Pituitary adenomas with invasion of the cavernous sinus space: a magnetic resonance imaging classification compared with surgical findings. Neurosurgery 1993; 33: 610-8.

7. Edal AL, Skjödt K, Nepper-Rasmussen HJ. SIPAP: a new MR classification for pituitary adenomas. Suprasellar, infrasellar, parasellar, anterior and posterior. Acta Radiol 1997; 38: 30-6.
8. Mortini P, Barzaghi R, Losa M, Boari N, Giovanelli M. Surgical treatment of giant pituitary adenomas: strategies and results in a series of 95 consecutive patients. Neurosurgery 2007; 60: 993-1004.

9. Sinha S, Sharma BS. Giant pituitary adenomas - an enigma revisited. Microsurgical treatment strategies and outcome in a series of 250 patients. Br J Neurosurg 2010; 24: 31-9.

10. Qi S, Li Z, Pan J, et al. Relationships between magnetic resonance imaging (MRI) features and texture of pituitary adenomas. Chin J Nervous Mental Dis 2007; 33: 548-50.

11. Wu ZB, Su ZP, Wu JS, Zheng WM, Zhuge QC, Zhong M. Five years follow-up of invasive prolactinomas with special reference to the control of cavernous sinus invasion. Pituitary 2008; 11: 63-70.

12. Gondim JA, de Almeida JP, de Albuquerque LA, Schops M, Gomes E, Ferraz T. Headache associated with pituitary tumors. J Headache Pain 2009; 10: 15-20.

13. Lundin P, Pedersen F. Volume of pituitary macroadenomas: assessment by MRI. J Comput Assist Tomogr 1992; 16: 519-28.

14. Stadie AT, Kockro RA, Reisch R, et al. Virtual reality system for planning minimally invasive neurosurgery. Technical note. J Neurosurg 2008; 108: 382-94. 